\title{
Smart Heart Disease Detection using Particle Swarm Optimization and Support Vector Machine
}

\author{
Dr. J. S. Awati ${ }^{1}$, Prof. S.S. Patil ${ }^{2}$ and Dr. M.S. Kumbhar ${ }^{3}$ \\ ${ }^{123}$ ETC Department, Rajarambapu Institute of Technology, Maharashtra, India 414415 \\ *Corresponding Author: Dr. J. S. Awati; Email: jayashree.awati@ ritindia.edu
}

\begin{abstract}
Healthcare and disease detection in early stage is important in every human being. Proper and optimum detection of disease with smart controller is done using Particle swarm optimization (PSO) and Support Vector Machine (SVM). The research includes the Fuzzy Proportional Integral and Derivative (Fuzzy PID) controller was used with support vector machine to classify the heart disease. Particle Swarm Optimization is designed to remove the noise introduced in Electrocardiogram signal. Fuzzy PID controller was implemented for disease detection and prediction. Fuzzy PID controller provides most accurate and stable results.
\end{abstract}

Keywords: Finite Impulse Response, Fuzzy controller, PID, Support vector machine.

\begin{tabular}{|c|c|}
\hline \multicolumn{2}{|l|}{ ARTICLE INFORMATION } \\
\hline \multicolumn{2}{|c|}{$\begin{array}{l}\text { Author(s): Dr. J. S. Awati, Prof. S.S. Patil and Dr. M.S. Kumbhar } \\
\text { Received: Dec 01, 2021; Accepted: Feb 01, 2022; Publis hed: Feb 10, 2022; }\end{array}$} \\
\hline $\begin{array}{l}\text { e-ISSN: 2347-470X; } \\
\text { Paper Id: IJEER120102; }\end{array}$ & crossef member \\
\hline $\begin{array}{l}\text { Citation: doi.org/10.37391/IJEER.090405 } \\
\text { Webpage-link: }\end{array}$ & $\begin{array}{l}\text { CROSSREFOORG } \\
\text { THE CTATION LINKING BACKBONE }\end{array}$ \\
\hline
\end{tabular}

\section{INTRODUCTION}

The Electrocardiogram (ECG/EKG) signal represented with respect to time and frequency are considered for detection for different heart disease. ECG signal is considered as excellent gadget for monitoring heart function. ECG signal provides the rate and beat of heartbeat of circulation system for evaluation. ECG signals are further evaluated by fuzzy PID controller for proper evaluation. The field of sign getting ready is a noteworthy field of study and one that makes possible various fields, for instance, exchanges Speech affirmation structures moreover correspondence programming necessity for examining and dealing with data to identify particular words in a verbally communicated sentence. Prosperity watching is by and by ending up being a bit of standard everyday presence. The current human administrations industry expects to give better prosperity organizations to people in a capable and patient sincere way.

There is a structure made for the cathode game plan for ECG. Ten cathodes are requiring to convey 12 electrical viewpoints on heart. The recorded signals are taking from each cathoderay electrode. In addition, this recorded sign is printed which is electrocardiogram.

Proper and optimum detection of heart disease with PID controller is done using support vector machine and particle swarm optimization. The Support Vector Machine was used to classify the heart disease. Particle Swarm Optimization is used for noise removal from ECG signal and Fuzzy PID controller was implemented for proper disease detection and prediction.
Fuzzy PID controller was used to provide most accurate and stable results.

\section{LITERATURE REVIEW}

Different procedures have been proposed and a thick composing is open for ECG signal embellishment and isolating. Regardless, all experience the evil impacts of different hindrances.

The researcher proved that ECG signals are used for exhibiting the patient condition and utilized for evaluation and treatments of various heart contaminations. They utilized complex QRS wave, $\mathrm{T}$ wave and $\mathrm{P}$ wave for assessing the sickness. The suggested to use $\mathrm{CNN}$ for recognizing distinctive heart disorders [1].

The authors used Support Vector Machine for filtering and feature extraction. They proved that modified disclosure and portrayal of upheavals can improvise the noise containing electrocardiogram (ECG). They also suggested that designed channels are based on ousting collectibles. Furthermore, the paper contains SVM estimation that includes a customized classifier to perceive the five pathologies [2].

The researchers presented ECG Data Acquisition system. They implemented 12 lead and presented a novel arrangement of an accumulate ECG signal information utilized to communicate customer' PDA by using Bluetooth for next planning, taking care of, and appearing. The three areas are combined together to achieve somewhat size, remote and flexible ECG acquiring structure [3].

Authors developed the system which can perceive the appearances of a heart stroke at a starting time and thwart it. It isn't serviceable for a normal man to routinely encounter extravagant tests prefer the ECG and in as such there ought to be a structure set up which is useful and strong, in predicting the chances of a coronary disease. The developed application can be useful the differentiation of a coronary sickness given central signs like age, sex, beat rate, etc. The artificial 
intelligence provides the most accurate and reliable system for the biomedical applications [4].

Researchers presented count for Heart Rate acknowledgment reliant on Short-Term Autocorrelation Center Clipping strategy. They used this count is for normal sign area, electrocardiogram, in uproarious condition with package of antiquated rarities. Using this estimation is in like manner possible recognize the $\mathrm{R}$ pointers in the PQRST complex of the ECG signal. They presented the new system for the beat changeability estimation. HRV module relies upon parametric and non-parametric techniques for the power apparition thickness estimation [5].

Authors presented different tuning approach for a flexible internal model-rule based sign unmistakable confirmation count whose computational costs are adequately low to allow a consistent utilization. The computation allows a quick Fourier rot of non-fixed signs that have an unequivocally obvious section [6].

Researchers utilized figures cutting edge sign isolating on electrocardiogram (ECG). Arranged channels are based on removing deftly sort out $50 \mathrm{~Hz}$ repeat and breathing muscle antiquated rarities. The quick systems for beat disclosure are ECG signal apparition stall Short-Term Autocorrelation technique many figuring's for beat ID rely upon QRS complex area and hear rate is figured like division between QRS structures [7].

Authors proposed an adaptable breaking point system on spectrogram enlisted using Short Time Fourier Transform (STFT) for QRS complex ID in electrocardiogram (ECG) signal. The computation involves pre-setting up the unrefined ECG sign to oust the electrical link check, enlisting the STFT, applying flexible thresholding strategy and followed by recognizing QRS tops [8].

In the paper, researchers presented a better methodology than exchange existing filtering strategy for curving free ECG signal. A belt-type ECG estimation system has been made to measure ECG signal in consistently life [9].

Researchers focused on electrocardiograph telemetry contraptions is proposed which surveys the idea of electrocardiogram signals picked up in solo circumstances, raises the insistence of the conveyed break down, and stimulates protective exercises when significant. The proposed count is realized in conditions when electrocardiogram signals are extraordinarily defenseless against antiquated rarities [10].

\section{BLOCK DIAGRAM}

The proposed work consists of two stages in which first stage consist of design of PSO filter for removing noise from ECG signal and second stage consist of disease detection using support vector machine classifier. Figure 1 shows the system block diagram and Figure 2 shows the fuzzy controller and Figure 3 shows the PID controller inside the fuzzy controller. Figure 1 shows the System block diagram which contains the
4 blocks, ECG signal acquisition, PSO_Filter to ECG signal, Fuzzy PID controller and Evaluation of disease.

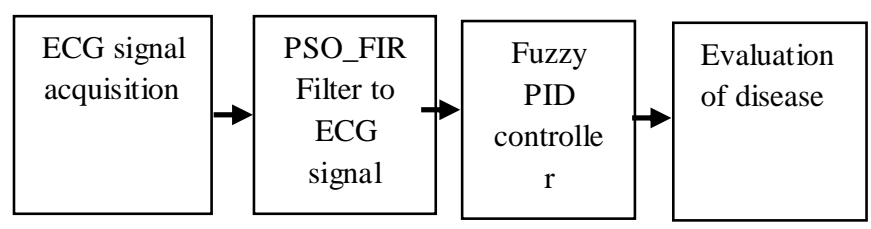

Figure 1: System B lock Diagram

ECG signal are acquired from electrodes. The noise introduced in ECG signals are removed by Designed PSO filter. PSO follows the same frequency as FIR filter for a specific coefficient and realizes the work as normal FIR filter with optimization. The comparison of PSO filter and FIR filter is done for cross validation of PSO filter with FIR filter. For research the high pass, band pass and low pass filters are designed with same coefficients and frequency response. ECG signal contain noise which must be removed. FIR filter removes the noise from ECG signal and fed this signal for further analysis. The complete noise signal should be removed from the ECG signal for medical diagnosis. Therefor the PSO FIR filter is designed to remove the noise from ECG signal.

$x_{n}=x_{n}+v_{n} * \delta(\mathrm{t})$

Where,

$x_{n}$ - Position of the n particle.

$v_{n}$ - Velocity of the $\mathrm{n}$ particle.

$\delta(t)$ - The time interval of every particle.

The Particle swarm optimization is given by following equation,

$\mathrm{V}_{\mathrm{i}}(\mathrm{t}+1)=\mathrm{w} \mathrm{V} \mathrm{i}(\mathrm{t})+\mathrm{c}_{1} \mathrm{r}_{1}\left[\mathrm{x}_{\mathrm{i}}(\mathrm{t})-\mathrm{x}_{\mathrm{i}}(\mathrm{t})\right]+\mathrm{c}_{2} \mathrm{r}_{2}\left[\begin{array}{c}g(t) \\ x_{i}(t)\end{array}\right]$

Where,

$$
\begin{aligned}
& \mathrm{i}-\text { Particle coefficient. } \\
& \mathrm{w} \text { - Internal coefficient. } \\
& \mathrm{c}_{1}, \mathrm{c}_{2}-\text { Acceleration coefficient, }(0 . \leq \mathrm{c} 1, \mathrm{c} 2 \leq 2) \\
& \mathrm{r}_{1}, \mathrm{r}_{2}-\text { Random value, }(0 . \leq \mathrm{r} 1, \mathrm{r} 2 \leq 1)
\end{aligned}
$$

The updated position using PSO is given by,

$$
\mathrm{x}_{\mathrm{i}}(\mathrm{t}+1)=\mathrm{x}_{\mathrm{i}}(\mathrm{t})+\mathrm{v}_{\mathrm{i}}(\mathrm{t}+1)
$$

The noise from ECG signal is removed using PSO based filter. The wavelet pyramidal decomposition method is used to extract wavelet features along with PQRST features of ECG signal. The dataset signals are also processed and features are extracted for training of SVM. The test input signal features are then classified using trained SVM model and display the type of disease. After noise removal the signals are applied to fuzzy controller. Inside the fuzzy controller the PID controller is used i.e., fuzzy PID controller is implemented. 


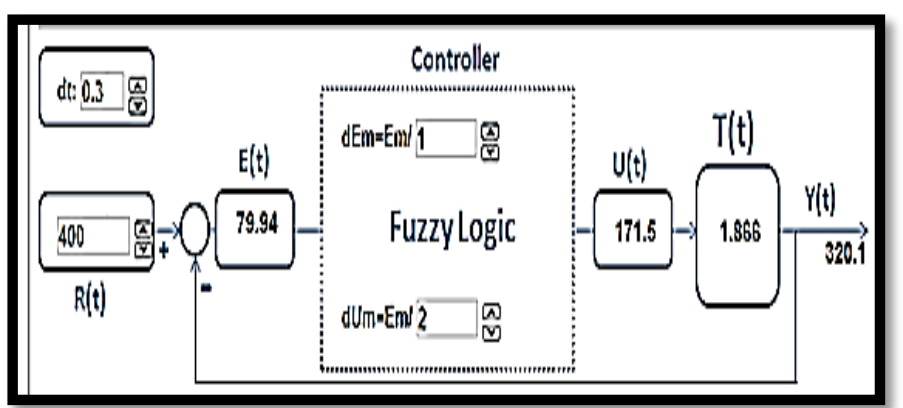

Figure 2: Fuzzy PID controller

Figure 2 demonstrates the fuzzy PID controller. Fuzzy controller contains the fuzzification, inference engine and defuzzification module. The inference engine used is Sugeno type inference Engine is implemented. The defuzzification method implemented is centroid defuzzification method.

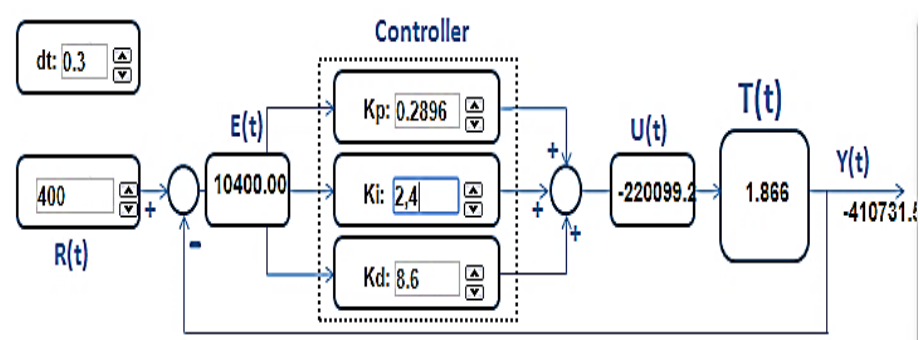

Figure 3: PID control inside fuzzy controller

Figure 3 demonstrates the fuzzy PID controller and PID control actions. In Fuzzy PID controller the following matrix is used.

$\mathrm{A}=[[-4,-3],[1,-8]]$ and $\mathrm{B}=[[1,0],[0,1]]$ and $\mathrm{C}=[1,0]$ is used for $\mathrm{T}(\mathrm{d})$.

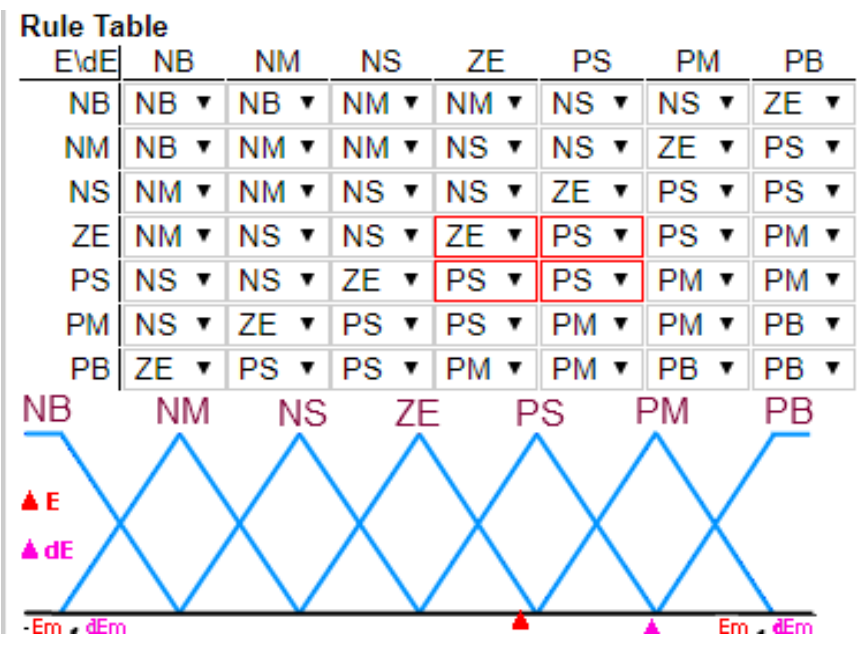

Figure 4: Design rule base for fuzzy PID controller

Figure 4 establishes the rule base system implemented in fuzzy PID controller. The linguistic hedges used are NB means negative Big and $\mathrm{PB}$ means positive Big. In this way $\mathrm{M}$ means medium, $\mathrm{S}$ means small and $\mathrm{Ze}$ means zero are used in rule base. As three variables are used in the fuzzy controller such as P, I and D controller inputs i.e., PID controller. The rules are written in this way. Total 49 rules are written which can be evaluated using rule base table.

\section{RESULTS AND ANALYSIS}

4.1 Input ECG Signal

The acquired ECG Signal from Physonet.org data set and used for further filter analysis. The Figure 5 shows the ECG signal containing Power line noise, Body contraction Nosie.

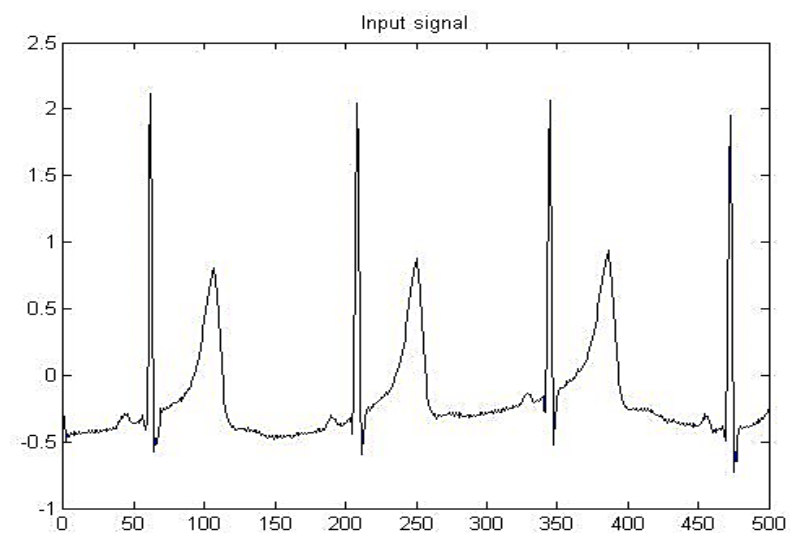

Figure 5: ECG signal acquired

\subsection{PSO Noise Removal}

The PSO optimization is applied on noisy ECG signal to remove noise and get clean ECG signal. The FIR filter complexity reduction for higher order filers is main objective for using PSO method. Figure 6 shows the filtering output of ECG signal.

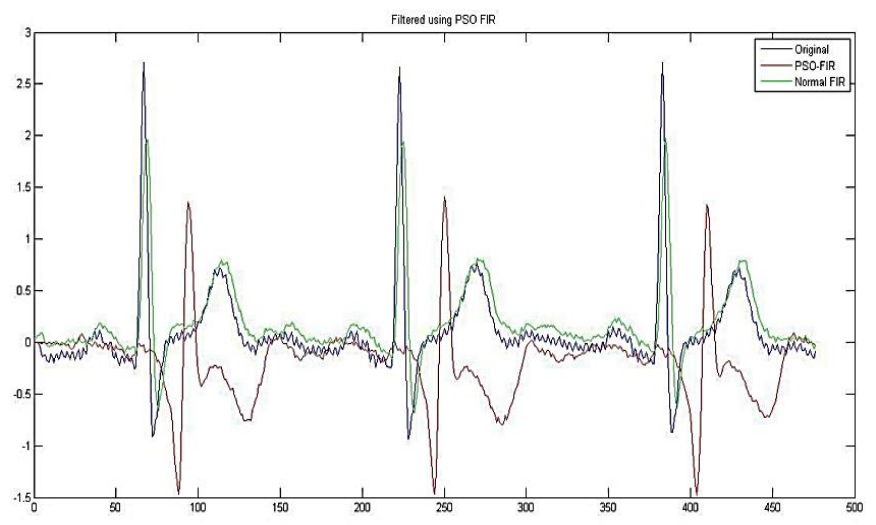

Figure 6: Noise removal using PSO based filter

4.3 Plot of (P, I, PI, PID) controller 


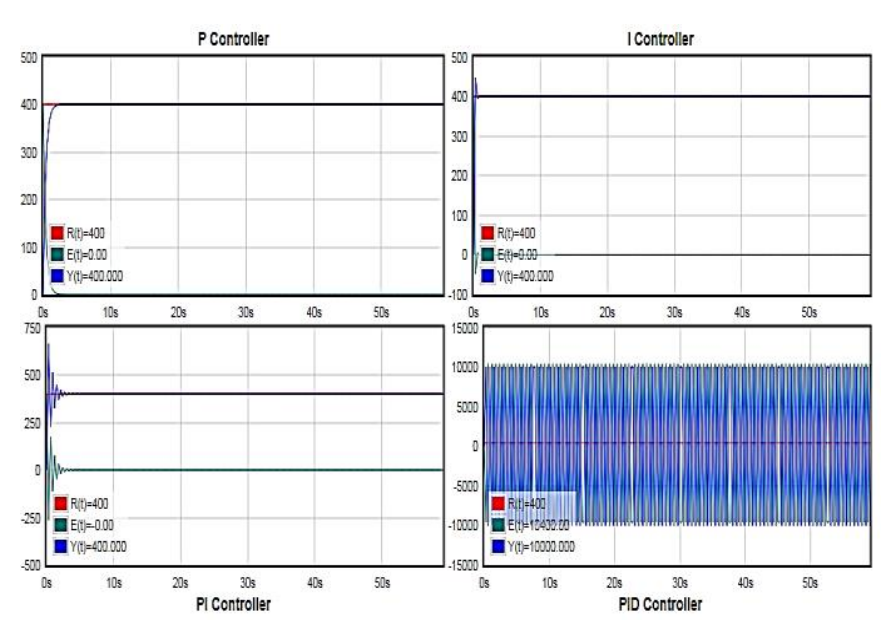

Figure 7: Plot of (P, I, PI, PID) controller

Figure 7 demonstrates the P, I, PI and PID controller values adjusted in fuzzy controller. PID controller values keeps the values stable and controlled output for disease prediction because it gives the controlled output signal with controlled set point. This is implemented in defuzzification in fuzzy controller. The defuzzification method implemented is centroid defuzzification method.

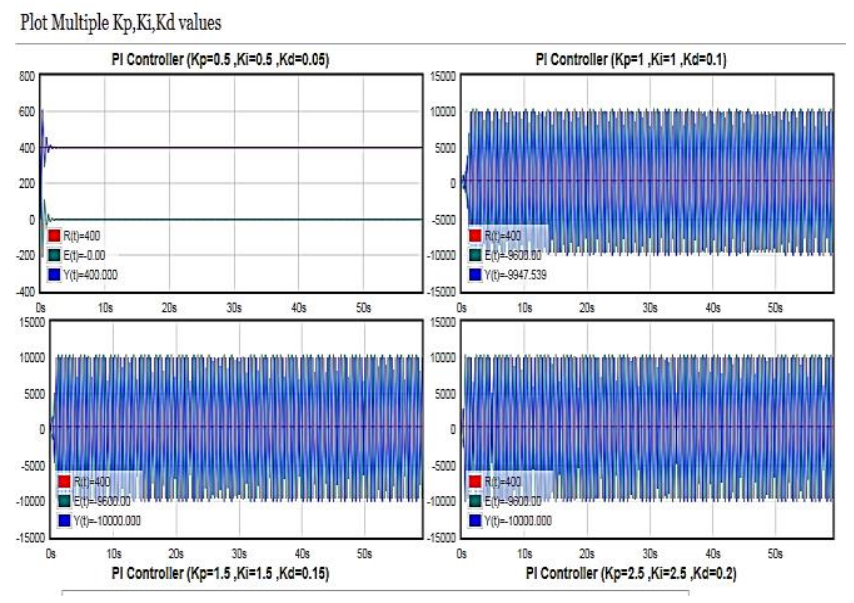

Figure 8: Plot of $\left(\mathrm{K}_{\mathrm{p}}, \mathrm{K}_{\mathrm{i}}, \mathrm{K}_{\mathrm{d}}\right)$ controller

Figure 8 shows the $\mathrm{K}_{\mathrm{p}}, \mathrm{K}_{\mathrm{i}}$ and $\mathrm{K}_{\mathrm{d}}$ values adjusted during the optimization.

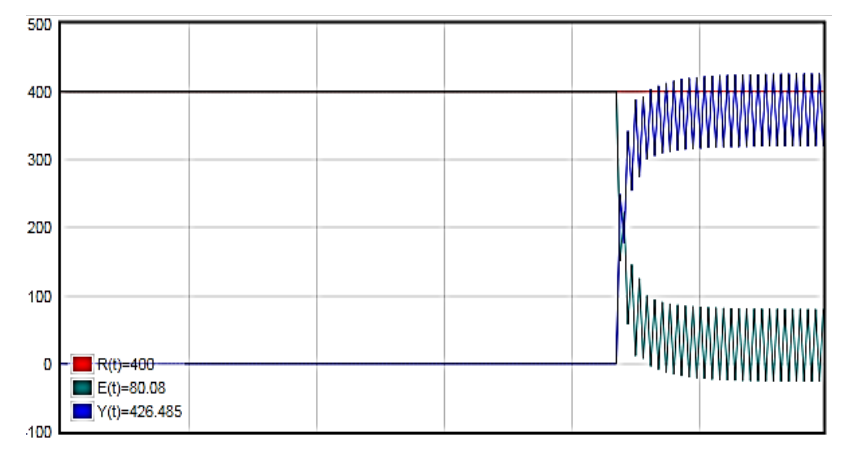

Figure 9: Plot of PID controller
The Figure 9 demonstrate the setting of PID controller values.

\subsection{Disease detection}

The figure 10 shows the ECG signal of human heart acquired form electrodes. The Electrocardiogram contain $\mathrm{P}$ wave, Q wave, $\mathrm{T}$ wave and $\mathrm{R}$ wave. The atrial depolarization of the electrical action shown by $\mathrm{P}$ wave. The SVM QRS complex used for the classification and extraction. $\mathrm{T}$ wave makes ventricular repolarization.

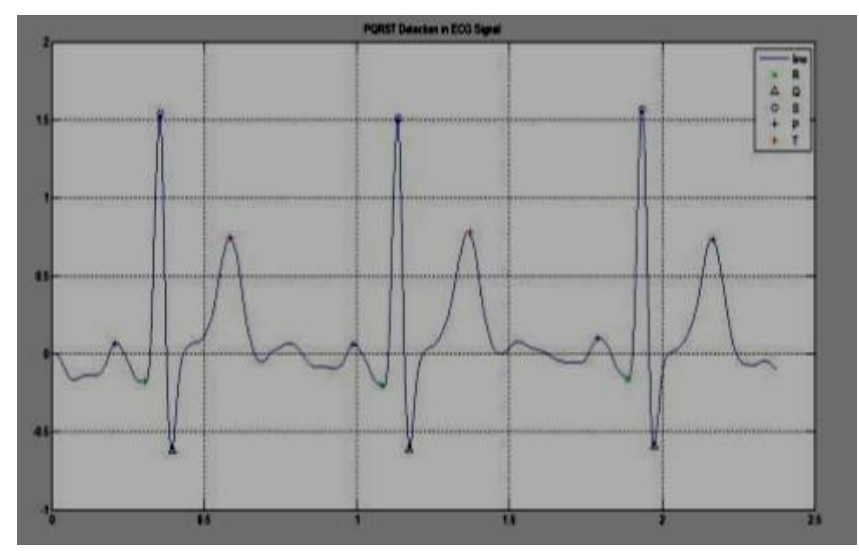

Figure 10: PQRST Features

$\mathrm{T}$ wave have longer range than the $\mathrm{QRS}$ complex wave. $\mathrm{R}$ wave is used for fixing the ventricular apex. Feature extraction ECG features extraction subject to ordinary equivalently as supernatural evaluation. Considering these centers various features are removed, for instance, beat, typical heartbeat, standard deviation in beat, between time in tops (R-R), and testing entropy.

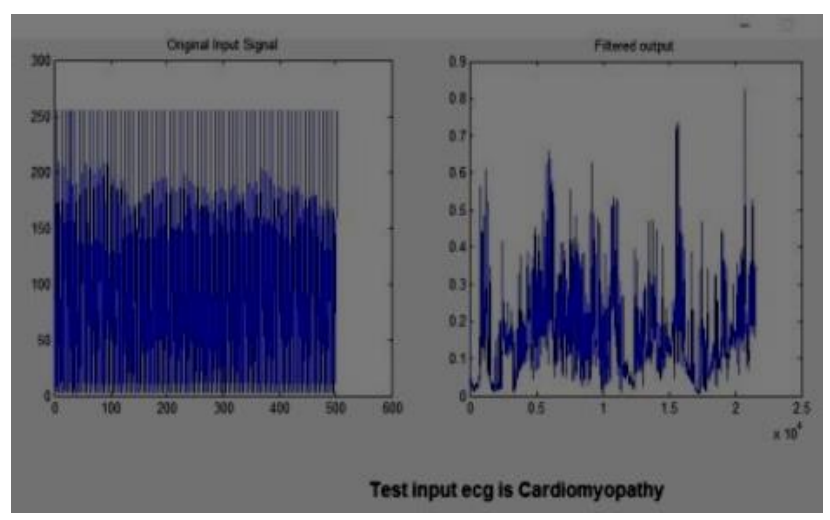

Figure 11: Disease detected output

Here, in this paper depicts the precision for five infections. The ailments are differentiated and different features type. The classifier first trains the data by then used for test which has cross endorsement by 10 wrinkles. The QRS is astounding sign present in the ECG waveform. This sign is erratically gathered into the ten subsections. The Nine subsections are used for the readiness reason and one is used for testing. In this portrayal contains different parameter, for instance, affectability, precision and expresses. This parameter is given underneath. 
Accuracy $=($ Positive True + Negative False $)+($ Positive True + Negative False+ Positive True + Negative False)

$$
\text { Sensitivity }=\frac{\text { Positive True }}{(\text { Positive True }+ \text { Negative False })}
$$

Negative False $)+($ Positive True + Negative False+ Positive True + Negative False)

$$
\text { Specificity }=\frac{\text { Negative True }}{(\text { Negative True }+ \text { Negative False })}
$$

The table 1 indicates the significance of Boolean values used for calculations.

Table 1: Significance of Boolean values

\begin{tabular}{|c|c|c|}
\hline Input & Detection & Category \\
\hline Disease & Disease & True Positive \\
\hline Disease & Normal & True Negative \\
\hline Normal & Disease & False Positive \\
\hline Normal & Normal & False Negative \\
\hline
\end{tabular}

\section{CONCLUSION}

In the ECG dataset contains 150 sample which has five types of diseases such as Dysrhythmia, Bundle Branch Block, Heart failure, Cardiomyopathy, Healthy control, and Myocardiallnfarction. The $96 \%$ accuracy gives the developed classifier. The support vector machine gives the accuracy up $96 \%$ on the basis of classification. The average detection rate also called as accuracy and achieved up to $98 \%$ using PID controller. The two steps have been done before calculating the accuracy such as removing the noise and calculating the features of ECG signal. To remove the noise from corrupted signal filters technics are used. The different type of filter is used for filter out the ECG signal. The process of features extraction is used to reduce components dimensionality. The features are clean and reliable. This is the remove the unwanted components. In this system because the support vector machine classifier and kernel parameter are work together. The cross validation is used to obtain the kernel parameter and constant. The fuzzy PID controller was used for disease prediction.

\section{REFERENCES}

[1] N. Gawande, A. Barhatt, "Heart Diseases Classification using Convolutional Neural Network", The $2^{\text {nd }}$ International Conference on Communication and Electronics Systems, 2017.

[2] N. V. Khandait, A A Shirolkar, "ECG signal processing using classifier to analyses cardiovascular disease", $3{ }^{\text {rd }}$ International Conference on Computing Methodologies and Communication, 2019.

[3] Cheng Sun, Jingsheng Liao, Gang Wang and Baopu Li, "A Portable 12Lead ECG Acquisition System", International Conference on Information and Automation Yinchuan, China, August 2013.

[4] A. Gavhane and G. Kokkula, "Prediction of Heart Disease Using Machine Learning", 2nd International conference on Electronics, Communication and Aerospace Technology, 2018.

[5] Piotrowskia Z. Rózanowski K. "Robust Algorithm for Heart Rate (HR) Detection and Heart Rate Variability (HRV) Estimation", ACTA Physica Polonica, vol. 118, pp. 131 - 135, No. 1, 2010.

[6] Chen, Jenn-Shyong, "Signal Filtering Using the Hilbert-Huang Transform", Journal of Science and Engineering Technology, pp. 75-84, March. 2010, 6(1).

[7] Surda, J. Lovas, S. Pucik, J. Jus, M. "Spectral Properties of ECG Signal”, 17th International Conference, Brno, Czech Republic, pp. $1-5,24$ th -25 th April 2007.

[8] Kohler, B.U. Hennig, C. Orglmeister, R. "The principles of software QRS detection", Engineering in Medicine and Biology Magazine IEEE, vol. 21, pp. 42 - 57, January -February 2002.

[9] H., Do-Un Jeong, "Development of Wearable ECG Measurement System Using EMD for Motion Artifact Removal", Dept. of Ubiquitous IT Engineering Graduate School, Dongseo University Busan,South Korea.

[10] Nygårds, M. Sörnmo, L. "Delineation of the QRS complex using the envelope of the ECG", Medical and Biological Engineering and Computing, vol. 21, pp. $538-547$, September 1983.

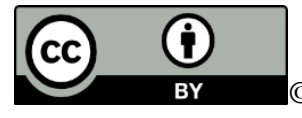

2021 by the Dr. J. S. Awati, Prof. S.S. Patil and Dr. M.S. Kumbhar. Submitted for possible open access publication under the terms and conditions of the Creative Commons Attribution (CC BY) license

(http://creativecommons.org/licenses/by/4.0/). 